\title{
Systematisation of seismic mitigation planning at urban scale
}

\author{
D.L. Viana, A. Lima, G.D. Carlos, F. Gomes \& M.R. Correia \\ Escola Superior Gallaecia, Vila Nova de Cerveira, Portugal
}

P.B. Lourenço

University of Minho, Guimarães, Portugal

H. Varum

University of Porto, Porto, Portugal

\begin{abstract}
This chapter explores the relation between the seismic mitigation planning that was developed to contribute to minimise effects at urban scale, which arise within the seismic impact. In this sense, it deepens the notion that interdependence, solidarity and cohesion of the different morphological constituents promote a better prepared built fabric, when facing catastrophic phenomena of seismic origin. Preventive approaches and reactive measures arising from the testimony of earthquake-resistant culture in Portugal are also mentioned. From the recognition of the importance of local know-how, infers a set of logical that evidence conditions to transcend the respective vernacular context, making it possible to generically systematize them.
\end{abstract}

\section{INITIAL REMARKS}

\subsection{Morphology and seismic-resistant culture}

The socio-physical organisation of groups and communities obeys to multiple factors that determine the place of settlements and the corresponding collective structure. Among them, issues such as access to resources, the availability of food (collected or grown / produced), storage and distribution - and consequent transport processes and exchange -, in addition to aspects related to the overall security and the convenience of natural conditions of the territories (its topography and climate, for example.), are commonly regarded as decisive for the continued stay of population groups. Nevertheless, in many settlements, these logics face somewhat uncertain predictable phenomena, more difficult to frame, but with consequences and direct and indirect effects of great impact, implying risks - sometimes - not calculated associated with natural disasters, e.g. seismic activity. When this occurs, it is usually exposed the vulnerability of the settlements, and the need to mitigate the resulting damage. In this regard, it is pertinent to ask and witness the ways in which the relationship between the morphology of settlements and seismology was being addressed in order to - in good time - take shape a greater awareness of the significance of the last over the first, not only in terms of its configuration, but also in regards of the possible preventive measures and reactive approaches.

\subsection{Seismic-activity: a certainty}

The settlements configuration may take a variety of forms, which, in many cases, show contrasts in their built up fabric, the corresponding circulation networks and activities / land uses associated with them. Other variables such as the size of the sets, the housing density, grain size and porosity of mesh, the cadastral organisation and the level and type of instalment, not forgetting the range of public and / or collective space, conform aggregation systems with less or more aptitude to deal with seismic activity. The resulting community living, consolidating practices of earthquake-resistant culture, focuses on the expansion of collective spaces, the standardisation of roads, the provision of reserve areas for covering individuals, the foreshadowing of repeated and arranged architectural typologies of a linearly compact way . These references exemplify preventive approaches and reactive measures systematised in the research developed on the structure of the settlements - and its corresponding aggregation - of the case studies discussed throughout the research project 'SEISMIC-V' - Earthquake-Resistant Vernacular Culture in Portugal. It is also important to reinforce the notion that, in multiple contexts of settlements, such as those in different geographies of the country, it is urgent to rescue this "collective consciousness" for such a significant issue, whose doubt is not whether it will happen, but when it will happen. 


\section{SETTLEMENTS AND THEIR CONFIGURATIONS}

\subsection{Compact settlements}

It is known that from the human conglomeration results organised settlements as compact settlements, due to the concentration of people, goods, resources, and built (infra)structures, in view to host community life, cultural ties, social cohesion, economic development, scientific knowledge and technical progress. These are sites and places with potential for the substantiation of solidarity and mutual aid logics, supported by the gathering of knowledge and experience feeding strategies aimed at predictable scenarios. Along the research developed under the project 'SEISMIC-V', the legacy of this type of configuration proved to be significant for the results obtained. Thus, with reference to cases like Lisbon and Vila Real de Santo António, it was revealed a framework of concerted actions initiated to prevent the impact of new extreme events, such as earthquakes. Either in Lisbon or in Vila Real de Santo António (Fig.1), it was detected - following the recent most striking seismic events - a concern for planning spaces based on a strict orthogonal grid, the enlargement of arteries (aiming at broader streets and not so clogged) providing escaping routes and reserve areas. These preventive measures were not felt only in terms of morphology of the space system; it also had effects on the typological composition of the building fabric, conditioning volumetric, homogenising proportions, aligning heights and establishing a closer typemorphological relationship (e.g. forcing a greater interdependence between the conformation of buildings and their articulation with the surrounding streets). From this range of practices, progress was made in testing a theoretical corpus that requires both, attention and further developments.
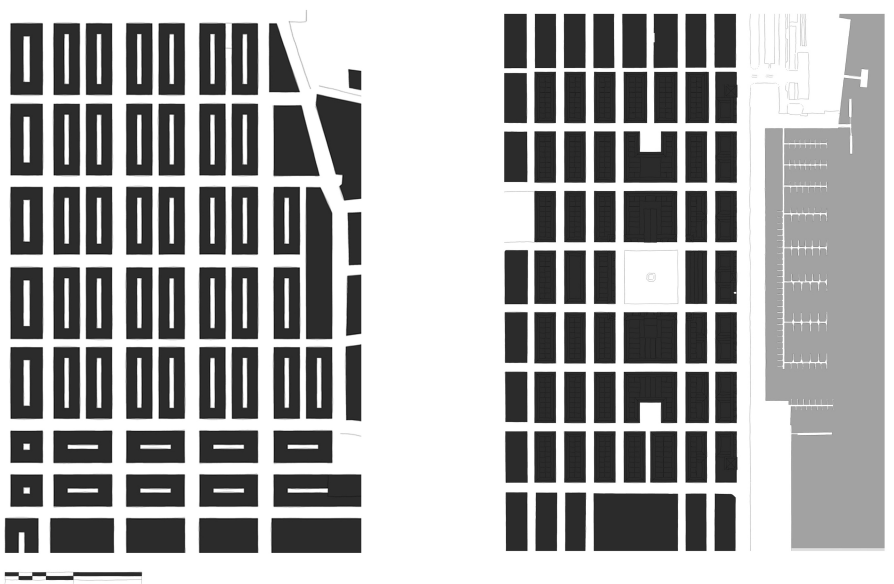

Figure 1. Orthogonal layout of the urban mesh in Lisbon (1) and Vila Real de Santo António (2) (credits: CIESG).

\subsection{Consolidated Centres}

The carried out survey also sought to focus on more empirical approaches to the problem of earthquakes. For this purpose, situations with "spontaneous" growth of settlements were taken into account, of which consolidated centres of organic matrix resulted, presenting morphological more intricate and deployed settings. The process setting of this kind of space system occurs in time and over time, wherein the construct leads to the accumulation density of the building in confined spaces and without previous planning. The scarcity of this planning increases the impact of seismic activity, also raising the potential for possible damage at the level of the structure and building fabric. Thus, Évora, Tavira and Lagos are selected for analysis (Fig.2).

Amongst these three case studies reactive measures to mitigate the effects produced by earthquakes are primarily verified. In opposition to preventive measures aforementioned - attempt to project the impact of future seismic events - the population implements (afterwards) a set of empirical solutions which aim to ensure the continuity and enhancement of existing constructions and the (infra)structures. These are logics that can be taken alone, or as a result of interventions that establish continuity between them, contributing to the overall solidarity of the settlements. Examples of this are actions on blocks, which are based on the physical cohesion of the same, promoting the distribution of counterarches, relieving arches, and vaults and braced links connections. These initiatives are relatively combined and (sometimes) of small scale become relevant when considered all together, implying individual or collective efforts of private and / or public character. This empirical and local know-how can play a key role as complement the general planning strategies.
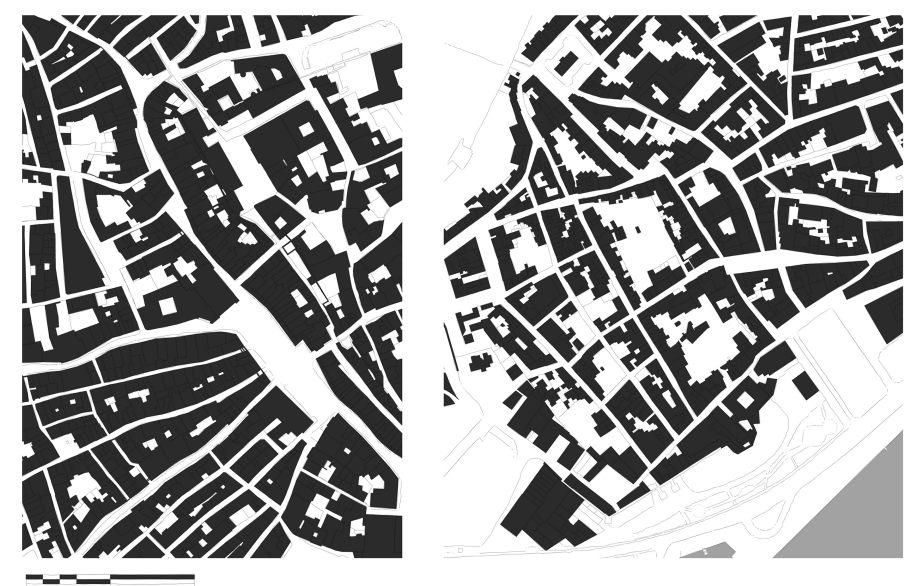

Figure 2. Historical Centre of Évora (1) and Lagos (2), consolidated centres of organic matrix (credits: CIESG) 


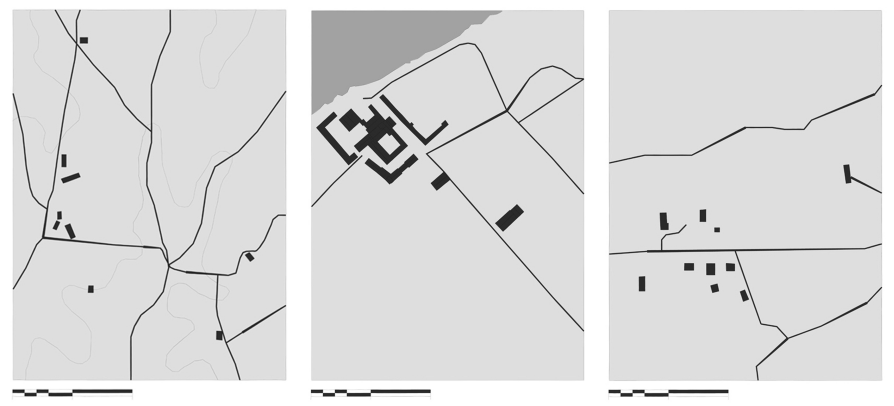

Figure 3. Settlement structure and built fabric. Trindade (1), Coruche (2) and Melides (3). (credits: CI-ESG
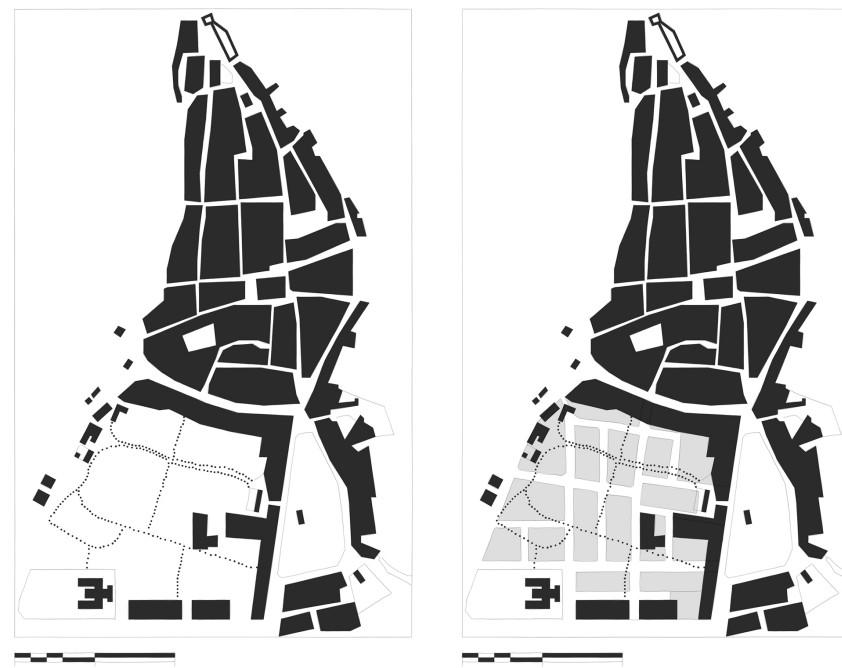

Figure 4. Benavente Plan, 1908 (1) and Plan and with the expansion area after the earthquake, 1909 (2). (credits: CI-ESG)

\subsection{Scattered structures}

The settlements with less continuous housing, morphological configuration were analysed. In the consistency of preventive approaches, even when generally seen, it was opposed a role of reactive measures. Considering it, in scattered structures, the empirical bias of the principles adopted anchored in the knowledge of the geo-morphological characteristics of the territory in which the settlements are distributed, an example being the use of geophysical features - such as rocky upwelling - for bases and Poiais (stone benches). When considering Melides, Coruche and Trindade (Fig 3), it is possible to specify actions, such as reconstructive reinforcement with earthquake-resistant elements based on various devices, such as buttresses and tie rods.

In the setting of scattered structures, there is yet an articulation between typological / tectonic logics and the specificity of the territory, its topography and location. The relationship between morphology and earthquake-resistant culture is in the deepening interdependence between natural and artificial systems that make up the settlements. The aloofness complicates the planning of preventive strategies scale and / or widely concerted, however, it does not decrease the density of the collective and shared knowledge as a result of trial and error processes, which should be systematised.

\subsection{Mixed Contexts}

In short, the settlements and types of forms indicated so far - based on what was said for the listed cases of study - exemplify aspects identified in the research that contribute to the generic definition of principles framed by a possible Earthquake-Resistant Vernacular Culture in Portugal. Noting the country, it is noticeable that it is composed of many free settlements, in which coexist the planned and the occupied, the rationality of the layouts and the organic nature of appropriate, the development of the (infra) structured and the so-called "spontaneous" growth. These are mixed contexts, whose morphology translates contrasts between collective policies and private initiatives, predictability of the land uses and actual activities, time and space, global agents and local actors of change, vigorous periods and recessionary times. The forms hybridisation mark these mixed contexts. In this framework, the research clarified that - in what case studies with this configuration matrix are concerned - the preventive and reactive measures to seismic activity (if any) share not only an empirical basis, but also theoretical one. As in everything else, the convergence of administratively planned strategies and the actions of people are everpresent.

Taking Benavente as an example, it may be said that these are combined in the different parts of the settlement: as regards with sectors said of "spontaneous" growth, of a more organic inclination (originating from earliest times), and in that, which relates to the areas of planned expansion for the resettlement of the population affected by the earthquake of 1909 (Fig.4). In these is the adoption of measures, such as the design of linear orthogonal meshes, the implementation of the enlargement streets and the establishment of blocks of symmetrical plant. The confrontation matrixes and the regular and irregular morphological elements amplify the situations and aspects to be resolved in earthquake-resistant terms, making it necessary a panoramically perspective appropriate indicators of a hypothetical Portuguese earthquake-resistant culture.

\section{BUILDING AND CONTINUOUS ROW SYSTEMS}

\subsection{Continuous or row buildings}

More than in morphological terms, the research has consolidated the perception that if there is - effectively - an Earthquake-Resistant Vernacular Culture in Portugal, it will have being much more structured around typological / tectonic aspects and aggrega- 
tion systems building, working towards the strengthening, cohesion and constructive solidarity of the fabric of the settlement and its adjacent space systems. It was evident that for the continuous and / or row building it is frequent the combination of aggregation systems, combining symmetric and regular plan constructions (with two orthogonal axes of symmetry) with earthquake-resistant elements, such as buttresses and the tie rods - not forgetting the aforementioned structural bearing walls (Fig 5). The presence of these elements are noticeable in places like Coruche, where are also detected other types of capacity improvement measures, poial situations and the blind arches. In this way it is sought to guarantee a greater stability and mechanical solidarity of the construction, aiming to improve the performance of the building for seismic demands, and reducing their impact.

\subsection{Isolated building}

The isolated construction is characterised - within the seismic context - by the homogeneous and compact configuration of the respective plan (often rectangular matrix), with a reduced (and small) number of openings, not forgetting the gabled roof and the chimney on the facade. The isolated building emerges as the most common type in the areas under study.

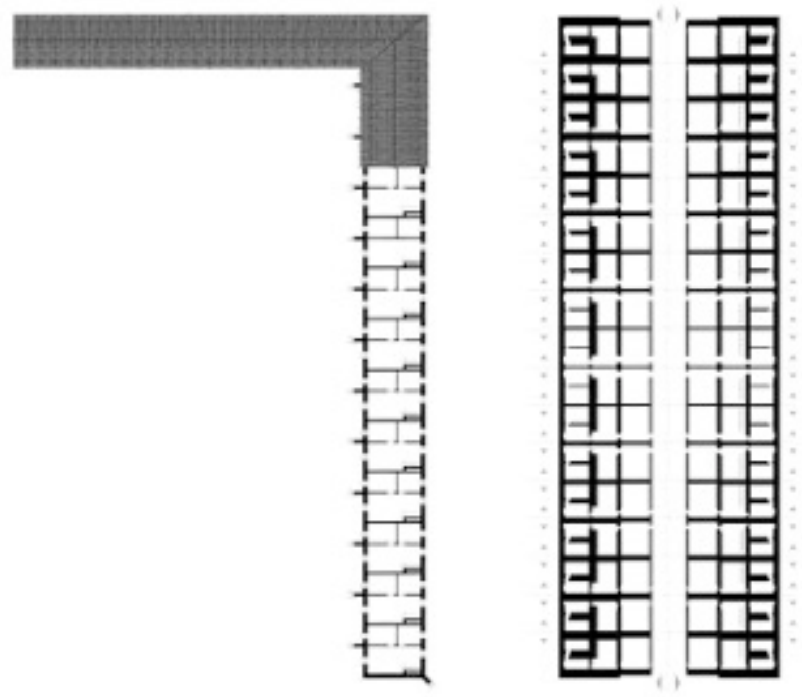

Figure 5. Row edification, Couche (1) Municipal district plan designed after the 1909 earthquake in Benavente (2) (credits: CI-ESG).

\subsection{Blocks}

The structure and the logics of aggregation provided by type-morphological devices, such as blocks, contribute to the diversity of the forms of the settlements. Nevertheless, the research conducted within the multiple case studies converge towards substantiate the notion that - for seismic demands context there are parallel alignments that form blocks of standardized plan, geometrically regular, and with an homogeneous architectural "language". In addition, aggregation systems building in symmetrical and longitudinal row are protagonists. Narrow streets or courtyards mediate the built fabric, providing small reserve spaces and / or relief.

The walls of separation between dwellings are also features, solidly built and with no span, looking for to ensure its maximum compactness and capacity. Further to this aspect, it is also to highlight the closed structures of the blocks and a consequent overall structural stability of the whole. Particularly in the case of Evora, it is to emphasize the occurrence of linkages between existing buildings (reactive measure, guaranteed by the use of counter-arcs) (Fig.6) and to promote a greater stability of the whole of the buildings based in mechanical principles continuity and lateral displacement control. Prevails the notion that the built fabric more than understood as the sum of buildings, should behave like an interdependent system, reacting as a whole to the impact of seismic demands, so that - from scale logic - it is established a "solidarity" and "cohesion" between the buildings, which allow them to be better prepared to withstand the effects of earthquake demands.

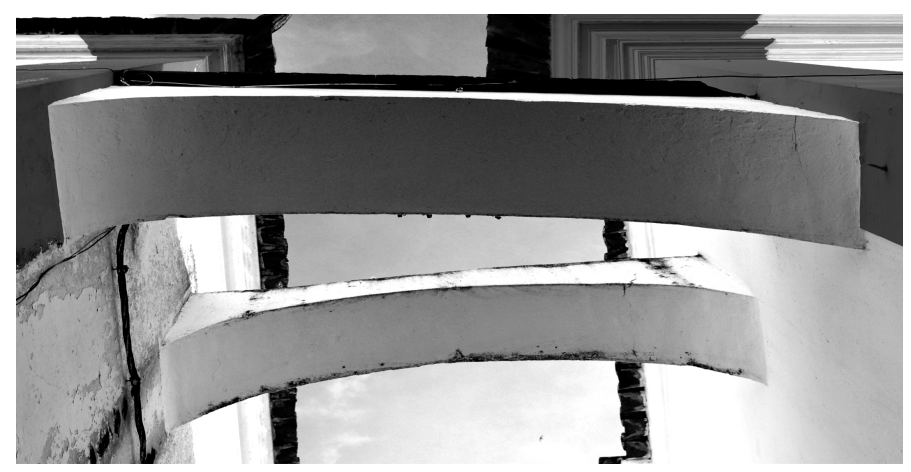

Figure 6. Historical centre of Évora, placing of counter-arcs between the different blocks (credits: CI-ESG).

\subsection{Residential blocks}

The result of the impact of seismic activity may have multiple consequences, since the collapse of buildings, construction debris scattering, declining of objects, obstruction of traffic routes, disabling (infra) structures, the collapse of bridges and viaducts, floods, and fires spread between buildings, etc. Many of the measures presented so far attempt to project / mitigate these and other effects of earthquakes. The residential blocks are the typological plans most likely to suffer from these and other damages (given the respective scale, number of floors, and number of dwellings). Seen it, the atten- 
tion to the residential buildings should be protagonist in seismic activity contexts. Given the relevance of this problem, along the research carried out, was considered a history of significant experiences that took place in Lisbon. Among them there are the walls separating property / plots of land (which rise beyond roof quota in order to prevent the spread of fire) and limiting the number of floors (in Lisbon, four below the cornice). But more important than was above mentioned, it's the conceptualisation of the built fabric based not in the individual logical entities, but divided into blocks (almost always of rectangular base), trimmed by a continuous front of streets. It is a solution with a better use of land and an urban facade that repeats the compositional effect of the urban design. It regularises views, clarifies the overall accessibility of the whole, contributing to the guidance of their users, also enhancing the spatial configuration of the unit system. These are aspects to be valued, in a post-seismic 'chaos'.

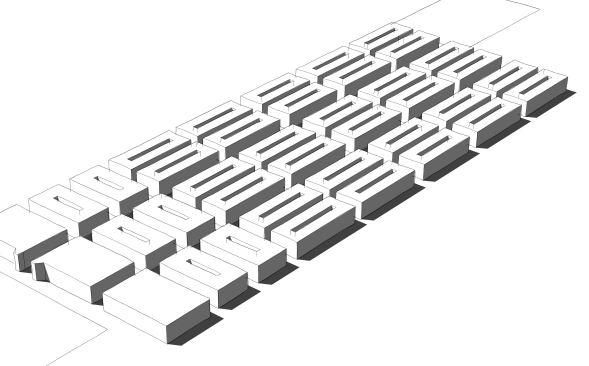

Figure 7. Orthogonal grid, N-S axis (direction of concussions), and spaces reservation, outlined in the proposal of Eugénio dos Santos and Carlos Mardel in Lisbon 1773. (credits: CI-ESG)

\section{PREVENTIVE AND REACTIVE MEASURES}

As already seen in seismic context, two types of actions taken by the people are to distinguish. A direct action, which aims to repair the damage already produced in building structures - a reactive approach - and another, which is indirect, aiming at to reduce damages in future occurrences - the preventive approach.

In terms of urban planning as a process that anticipates the structuring of a given territory, the adopted measures are preventive in nature, such as Lisbon, Vila Real de Santo António and Benavente.

The reconstruction plan for Lisbon, drawn up in 1756 by the engineer Manuel da Maia, established a series of "new urban concepts" that became a reference. The first largely measure, adopted in the full organisation of the urban fabric, was the orientation of the blocks. The base structure of the building fabric would adopt a north-south direction, the main orientation of concussions, so as to, considering the conservation of energy rule, only the smaller facades would be exposed to the projection of seismic waves (Mascarenhas, 2009).

The geometrically structured and linearly regular urban grid designed to Lisbon, defined by rigorous and orthogonal alignment of facades, allows a quick and orderly evacuation system in case of earthquake (or fire). At the extremities of the block were implemented two large squares, as reserve spaces, the Commerce Square (south) and the Rossio Square (north) (Fig.7).

The buildings were grouped in closed blocks, so as to give greater structural stability to the whole. This would reduce the number of gables subject to shocks, reducing and restricting the rotation capacity of each urban element / building (since each would prevent the rotation of the other). Another of the great measures of reference, established by the Plan of Lisbon, was the widening of the streets: the main would have 60 spans (with a lane for cars with 50 spans and another one of 10 spans on each side for pedestrians); secondary streets would have about 40 spans.

The rule of proportion arises also as a reference element; i.e. based on the ratio between the width of the streets and the height of buildings, all blocks have the same symmetry in doors, windows and arch centrings. This space limitation would diminish the risk of collapse over the streets.

Any compositional elements outside facade plan were also banned, such as steps, windows or door protections, rings, etc., as well as the openings near the wedges - as these weaken the structural continuity of the built blocks and other equipment. The urban guidelines established by the Engineer Manuel Maia for the reconstruction of Lisbon were the basis for the planning of new urban structures, as it is the case of Vila Real de Santo António. The plan outlined in 1773 by the architect Reinaldo Manuel organises that city according to an orthogonal grid (north-south, parallel to the direction of concussions) and with the same width of streets. The urban fabric would be defined by blocks of 240x100 spans, one and two stories high (Lopes et al, 2004).

And such as Lisbon, Vila Real de Santo António would enjoy also a "reserve space": the Marquês de Pombal Square, located at the midpoint of the space system.

Benavente, presents itself as mixed context, marked by the existence of two distinct urban plots: one is part of the Old Town; the other part is composed of the extension area (post-earthquake, 1909). After the 1909's earthquake, it was designed a General Plan for Roads, specifically a Cadastral Plan of the Village, indicating the new measures to apply to the existing layout (Old Town), and the structuring of the new extension 
area. As for the Old Town it was proposed an enlargement and a process of correction of the communication routes. The New Town, an area of expansion, was structured according to a rationalist matrix, where large wide streets prevailed, based on an orthogonal logic. The matrix was composed of uniform and aggregated blocks in symmetrical row houses, in two parallel alignments, separated by an inner street (constituting the set of preventive measures taken). These were also implemented in Benavente, a series of reactive measures on the building fabric, incorporating earthquake-resistant elements applied to the built area, highlighting given to the tie rods and tethers (Vieira, 2009).

Consolidated urban centres, such as Évora and Lagos, are associated with reactive approaches. Évora features a matrix of medieval origins, which marks the consolidated historical centre, consisting of blocks of compact 'spontaneous' growth. The city is characterised by the one-off introduction of seismic reinforcement elements as counter-arches, buttress reinforcement at the tops of the arcades. These elements are intended to provide greater structural stability to all blocks. In the case of Lagos, the introduced approach ensures the same standards, that is, a consolidated building fabric, where blocks marked by introduction of protruding elements such as continuous basis (whose function is to reinforce the volume) emerge.

In dispersed settlements, generally of rural character, reinforcement reactive measures of greater implementation are: the perimeter lashing strap (linking several connected built structures) and the buttress, which appears, sometimes, in the angles of the extremities of continuous buildings.

\section{FINAL REMARKS}

The shape and the organisation of the analysed settlements undoubtedly refer to the state of general destruction, verified after the events of greater impact. It is in the context of higher occupational density that this assumption becomes clearer. The triggered action is closely related to the analysis of the regenerative capacity of the affected structures, and to solve their technological constraints. In cases where a positive assessment is obtained, as Tavira and Evora, collective processes of spontaneous nature are triggered up, assuming the reconstruction almost immediately after those events. In these cases there is no apparent rupture between local constructive cultures and the reconstruction techniques applied. The built typologies and the techniques employed, which showed reasonable resistance, thus justify its perpetuation. Naturally, there is not a redefinition of the pre-existing urban fabric logic. The intervention occurs only at the one-off articulation level of existing structures, usually in what the reinforcement or on mooring adjacent elements is concerned, aiming at to maximize the structural solidarity of the whole. In these situations, the arcades, the counter-arches and the masonry vaulted structures are the most frequently used elements.

Considering more severe situations, and whenever it becomes mandatory to maintain the geographical position, it is witnessed a concerted action, coordinated by the administrative services. The planning of the urban form arises logically associated with a classical and formal architecture. Its great principles relate to the regularity of the layout and the systematization of shapes and construction techniques. The reconstruction plan of Lisbon downtown arises naturally as the main reference in these circumstances. The layout, the aggregation system, the architectural typology and construction processes are imposed, by force of circumstances, to the target population. This is the case of Vila Real de Santo António and the relocation neighbourhoods Benavente. Both adopt the urban model of regular orthogonal grid and assume the extension of the existing roads, ensured by the new deployment areas with suitable topography election. In these situations it is clear a larger aloofness between the typologies and techniques applied, and those of local nature. It must be emphasised though, a third context, which can be interpreted as a hybrid approach to the previously identified situations. In certain contexts, where the population is more distributed and the effects are not so focused, it is witnessed what appears to be a gradual cultural contamination. In this situation the application of models is replaced by the one-off incorporation of strategies or elements under the filter of regional culture. The melting of techniques and procedures thus contributes to the development of combined characteristics, which will gradually consolidate, as shown, for example, in the cases examined in Álcácer do Sal and Coruche.

\section{ACKNOWLEDGMENT}

The authors gratefully acknowledge the support by the Portuguese Science and Technology Foundation (FCT) to the research project 'SEISMIC-V - Vernacular Seismic Culture in Portugal' (PTDC/ATPAQI/ 3934/2012).

\section{REFERENCES}

Mascarenhas, J. (2009). Sistemas de Construção - V: O Edificio de rendimento da baixa pombalina de Lisboa. Técnicas de Construção. $3^{\mathrm{a}}$ Ed. Lisboa, Portugal: Livros Horizonte.

Lopes, M. S., Bento, R. \& Monteiro, M. (2004). Análise Sísmica de um Quarteirão Pombalino. Lisboa, Portugal: IST.

Vieira, R. (2009). Do Terramoto de 23 de Abril de 1909 à Reconstrução da vila de Benavente - um processo de reformulação e expansão urbana. Bevanente: CMB. 